\title{
Research on Intelligent Learning APP in Junior English Vocabulary Teaching
}

\author{
Ling Wang \\ School of Foreign Languages, Nanchang Normal University, Nanchang, China
}

\begin{abstract}
Vocabulary, as one of the most difficult problems for students and teachers, is also one of the most important elements in traditional teaching. This thesis is based on the problems existing in traditional vocabulary teaching and under the guidance of the mobile learning theory, the constructivism theory and the humanism theory. This thesis researches the application of intelligent Learning APP in Junior English vocabulary teaching. The research shows that the application of intelligent Learning APP, as one of the products of modern science and technology, is applied in Junior English vocabulary teaching. It effectively combines after-class teaching with in-class teaching, pays attention to students' individuality, improves teachers' teaching quality and reduces teaching pressure. The application of intelligent Learning APP has made up for the shortcomings of traditional teaching, stimulated students' interest in learning and made the teaching process more interesting.
\end{abstract}

Index Terms - intelligent learning APP, English vocabulary teaching, research

\section{INTRODUCTION}

\section{A. Significance of the Study}

The combination of information technology and education is not only appearing abroad but also developing gradually at home. Information technology lives with our life under the rapid development of information technology. Especially the mobile phone, it can be seen everywhere, like at our home, on the subway, on the road, etc. And everyone is able to use it from students to adult. Because of this mature technology, it will gradually combine with education. The world's trend of modern science and technology in primary and secondary schools in the 20th century has also brought a certain wave to our country. In "the national medium-and long-term education reform and the development plan" (2010-2010), it pointed out that information technology has a revolutionary impact on education development and must be given high attention. Under the instructions issued by the central government, the whole country is making great efforts to reform primary and secondary education by introducing multimedia and Internet into schools. Then, at the Fifth Plenary Session of the 18th Central Committee, it was further proposed to implement the "Internet plus" Plan and the innovation-driven development strategy (Wu Sufang, 2017). Nowadays, mobile phones are not only used for communication and play, but also for learning (Pimanmas \& Ninsuwana, 2015). Now many smart learning apps are continuously developed to meet the needs of education and students. The most important thing in the application of smart phone APP is the development of science and technology on English teaching in Junior Middle Schools. Many schools have perfect conditions to have computers; multimedia and wireless networks covering the campus. The second is the development of APP. Mobile phones are no longer limited to making phone calls and sending short messages, but also become your wallets, certificates, navigation, etc. When you go out, you all need is to bring a mobile phone, and you don't have to bring any extra things. Now the mobile phone can also be a learning platform instead of the traditional education (Liviu Constantin Stan, 2014). It doesn't need a fixed time and place that make learning more convenient. At the same time, it also can be used in students' spare time after class.

For students, using smart phone APP also has great benefits. First of all, it is conducive to improving their interest in learning (Gulcin Nagehan Sarica \& Nadire Cavus; 2009). Through the use of intelligent learning APP, it brings a kind of freshness to students, and students' ability to accept new things is better. The Box Fish APP contains a large number of animations and pictures, and knowledge is also connected with the latest development of the current network. Students can not only enjoy vivid and rich explanations, but also come into contact with fashionable things during the learning process. Secondly, the individuation of students is satisfied by using intelligent learning APP. When students use Box Fish APP, they can design the difficulty of learning and strengthen the weakness of their knowledge according to their basic abilities. This is different from the traditional teaching, and it also achieves the teaching effect of teaching students according to their individual needs. Then, using intelligent learning APP can strengthen the communication between teachers and students. Intelligent learning APP is based on mobile phone design which naturally includes language communication. Students can timely use the Box Fish APP to reflect their shortcomings to the teachers, and the teachers will give corresponding opinions after receiving the students' questions or reflections. The advances in information technology have led to a major shift in traditional education. In these transformations, the teaching in the information environment will pay more attention to the interaction and communication between teachers and students, students and students, and will focus on the individual differences of students (Lv Wanlu, 2017). 


\section{B. Research of Intelligent Learning APP at Home and Abroad}

Research on mobile learning around the world has a lot in common, that is, mobile learning can form a ubiquitous learning environment, which is more convenient for the development of mobile learning. And facing adult education, the concept of lifelong education has been further developed. Time for TIGER to ROAR! Technology Informatics Guiding Education Reform (O'Connor Siobhan \& Hubner Ursula \& Shaw Toria \& Blake Rachelle \& Ball Marion, 2017), the Technology Informatics Guiding Education Reform was established in 2006 in the United States and it integrates informatics competencies into nursing curricula and life-long learning. Researchers from Stanford University in the United States have studied the impact of mobile device application and teaching. The results show that using mobile phones for small tests is more effective than not using them.

In the contemporary society with rapid development of science and technology, mobile learning will also face some difficulties and challenges. For example, the network infrastructure cannot meet the needs of mobile device development. The development and implementation of the mobile teaching system is still a challenge for educators, and it is still difficult to develop the mobile teaching system, which can be compatible with any equipment because there is no perfect network standard.

In 2000, research on mobile learning began in China. Ding Xingfu translated Desmond Keegan's article, which is the first article on mobile learning in China. Subsequently, more and more researches on mobile learning have been carried out in China, and the researches are becoming more and more abundant. Zhang Jijun and Li Jianzhen (2010) mentioned that according to the characteristics of autonomous learning and mobile learning and the advantages existing in the self-learning mode, mobile learning equipment will help learners to study better in independent learning in the information age. Participation is the most important characteristic of students in interactive teaching. The exchange, sharing and communication of knowledge and experience between teachers and students, and between students and students are the most important. Teachers are no longer the only source of teaching information and the authority of knowledge, and students become masters of learning. Between teachers and students, students can exchange information and emotions through discussions and exchanges, which will give each student the opportunity to express his ideas. But also make the students who did not like to express themselves gradually integrate into this kind of active and interactive classroom atmosphere, which is helpful for students to improve their learning ability and expression ability.

\section{RELEVANT Theories About INTELLIGENT LEARNING APP}

\section{A. Brief Introduction of Mobile Learning Theory}

Mobile learning is based on digital learning and uses mobile devices to learn. Mobile learning is different from traditional learning. Teachers and students are all mobile by using intelligent learning APP. Mobile learning meets this requirement that is light and portable with flexible learning methods. Students can choose their favorite teachers to study in any environment by using mobile devices and downloading learning software. Second, we have the knowledge to keep pace with the times. This is an era of information explosion. Information is rapidly transmitted around us. The traditional teaching method cannot incorporate new knowledge until books are updated. However, book updating is an extremely complicated matter, and books themselves have certain authority and will not be changed at will. What we can learn from the book is limited. In this way, students will lose a lot of background knowledge when they study. Third, mobile learning reduces the costs to a great extent. Software can be installed on smart phones to listen to the lectures. Famous teachers from all over the world can teach through the online class. Students do not need to go to the classroom and site to listen to lectures like traditional teaching. This reduces the distance in space, not only at home but also abroad. Therefore, the use of mobile learning has become the most innovative learning method of the times.

\section{B. Brief Introduction of Constructivism Theory}

In constructivism theory, learning is not to transfer knowledge from the outside to memory, but to construct new understanding on the basis of original knowledge through the outside world. Constructivism theory is the basis of people's understanding of things, and its core is taking students as the center. Different from the traditional teacher-centered spoon-feeding learning that teacher only needs to play their own roles in the classroom and shows the knowledge in textbooks to students through blackboard writing or multimedia, ignoring the acceptance of the students. Under the guidance of constructivism theory, teachers, as a guiding part, give students the space in the class, stimulate their learning motivation through various methods, and let the students learn actively. Furthermore, constructivism theory expresses that students use learning materials to acquire knowledge under certain situations, which is consistent with our intelligent learning APP. It provides a certain learning environment for students, enables students to learn autonomously under the guidance of software, and focuses on the needs of students. Students can choose the courses they like and guide students from easy to difficult step by step. And in the process of learning, the new and old knowledge will be linked together to form a coherent learning. In the process of learning, difficulties will be enlarged and repeated, which is helpful for students to understand and digest.

\section{Brief Introduction of Humanistic Learning Theory}

Maslow, as the founder of humanism, fully affirms human value and dignity and actively advocates the realization of 
human potential. Humanism teaching thoughts not only focus on the development of cognition in teaching, but also pay more attention to the development rules of students' emotion, interest and motivation in teaching, and the understanding of students' inner psychological world. The intelligent learning APP conform the differences of students' interests, needs, experiences and personalities, develop students' potential, stimulate the interaction between their cognition and emotion, and pay attention to the restriction of psychological aspects such as creativity, cognition, motivation and emotion on behavior. It continuously confirms the learning status of the students during the learning process. If learning is too difficult, they will rearrange and analyze the difficulties in detail. And in the better place of learning, based on humanistic theory, intelligent learning APP will have corresponding encouragement mechanism, so that students can feel happy in the learning process and pay attention to their personal physical and mental growth.

\section{Problems Existing in English Vocabulary Teaching In Junior MidDle School}

\section{A. Single Teaching Method}

Vocabulary, as the basis of listening, speaking, reading and writing, is also the most important part of English learning (Brooke Dinsmore, 2019). But how to memorize words easily is still a difficult problem for Junior Middle School students. Most teachers only teach the words appearing and not related to students' life in classroom teaching. They should appropriately expand some words that students are interested in, and teach a little extra-curricular related content. In conventional method, it is not easy to arouse students' thirst for knowledge, but also does not reflect the spirit of teaching students in accordance with their aptitude, making students' vocabulary learning boring and monotonous. And in the teacher's subconsciousness, the more words the students learn, the better for the progress of English. In ordinary English teaching classes, there is often no time to help students memorize words, so students have to spend more time to recite them after class. The foundation of students' English ability is very different. The singleness of teaching materials also restricts the expansion of students' knowledge and the input of language information. Language learning is a cumulative process, which requires a large amount of extra-curricular reading to accumulate knowledge. The current English teaching is still stay in the syllabus, resulting in students' English reading is far from enough.

\section{B. Lack of Good Context}

Learning a new English word is not only the pronunciation and meaning of the word, but also the context in which it is used. Moreover, a word usually has many meanings and parts of speech. It is often difficult for students to notice the part of speech of new words in class, and teachers also ignore the teaching in this aspect. Usually, the best way to learn a word is to learn its usage in the corresponding articles, and to learn how to use it when learning. Teachers should also pay special attention to the connection between words. Learning is to extend new knowledge from old knowledge, and students often cannot think of this aspect, so teachers should give students good guidance in this aspect. For example, two long and similar words do have completely different meanings and two words with the same pronunciation but different spellings. In fact, in many cases we usually use Chinese to translate a word, but some English single words with similar meanings often have great differences in the use of context. Therefore, when teaching words, we can try to explain a word in English at this time. This is the most appropriate method.

\section{Deficiency of Organized Memory}

In traditional teaching, students seldom really like learning English. Most of them are sitting in the classroom without knowing why they study. Therefore, learning English will be more tiring for them. Memorizing words is a long-term process, which requires continuous review. However, junior middle school students lack motivation to learn, and they seldom take the initiative to review without the supervision of parents and teachers. In this way, it is easy for students forget English vocabulary. In addition, it is very difficult for students to memorize words just by spelling words. Students should find a suitable way to spell words by reading and writing phonetic symbols, and associate the phonetic symbols with the spelling of words. This is also something the teacher will not explain in detail in class. Teachers will ignore linking words with real life when teaching words, so students can't naturally associate words when memorizing words, making it not easier for them to remember words.

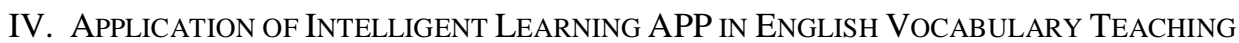

\section{A. Application of Box Fish APP before Class}

\section{Associative Memory}

The students open the Box Fish APP, choose the course that matches them, and begin to learn new words. In the learning part of the words, Box Fish APP takes the form of both pictures and texts. Next to each word, there is a picture that allows students to combine it with the picture when memorizing words. According to scientific evidence, the right brain of the brain has better memory than the left brain, while the right brain has better memory of images and the left brain has better memory of words. Therefore, this way of memorizing words can make it easier for students to remember words. At the same time, it also makes the process of memorizing words less boring, just like watching their favorite cartoons. For a word, Box Fish APP will not only display the part of speech of the word, but also be equipped 
with pictures.

2. Emphasis on Practice

In terms of memorizing words, students have learned new words, and then Box Fish APP has designed the words spelling, the oral practice and the sentence filling. Spelling helps students become familiar with words and remember them again, and at the same time, they can find out the words they have forgotten. Oral practice helps students to master the pronunciation of each word. Through memorizing words with pronunciation, students review the words again. In sentence filling, students can put a word into a sentence after the previous mastery of words. This is a gradual process from shallow to deep, simple to complex, so that students can better learn the words. By using Box Fish APP, students can not only remember and associate the words through pictures, but also deepen their understanding and use of words through the small games designed later. Let students learn more effectively.

\section{B. Application of Box Fish APP during Class}

\section{Game Design}

In class, teachers can use Box Fish for projection teaching, showing the pictures of words that students have memorized again, and students can associate words according to the pictures. And the teacher can design a group competition game here and students make a quick answer. In this way, it can not only help students review new words, but also improve their learning enthusiasm. Then, the students will focus on the words that are difficult to remember. Then the teacher can choose some difficult words from the new words to ask the students to make sentences, and examine the degree of students' mastery of the words, so that the teacher can not only explain the usage of key words, but also help the students create contexts.

2. Cultural Infiltration

Language and culture are inseparable. Without knowing a nation's culture, it is impossible to accurately master and use the nation's language. However, in the classroom, teachers often do not attach importance to the cultural meaning of English vocabulary, and students know little about the culture of British and American countries, resulting in students' little understanding of the learning content. Teachers can also use Box Fish APP to show students relevant cultural background videos, which helps students to understand foreign culture, and the different expresses between foreign and domestic cultures. At the same time, these interesting short videos will also enable students to deepen their understanding of cultural differences and let them feel the cultural atmosphere while studying. It also adds a little interest to the boring learning process.

\section{Application of Box Fish APP after Class}

\section{Scientific Memory}

According to the theory of The Ebbinghaus Forgetting Curve, the learned words will be forgotten with the passage of time, and the forgetting process is uneven. At present, teaching time is limited in the class, and the teachers cannot lead the students to review in the class. The students themselves have no clear plan after class, and the review time and method are very casual. According to the conclusion of the forgetting curve, the determination of the review point of the forgetting curve, the memorization method of the forgetting curve and the review process of the forgetting curve can benefit and improve human memory efficiency. The English word learning APP automatically arranges the cycle review according to the Ebbinghaus forgetting curve and the user's familiarity with words, and sets up interesting word memorization games to help the user memorize English words.

\section{Efficient Management}

After class, teachers can use Box Fish APP to assign homework, according to different learning situations of students, as appropriate. This not only takes care of the students' different basic level, but also achieves a good teaching effect, and at the same time reduces the students' learning burden. The students can finish the homework assigned by the teacher after class and review the words again at the same time. Many students cram for the exam each time and start memorizing words. At the end of the semester, the amount of that word is extremely large, and it is difficult for students to review. However, on the Box Fish APP, the learned words will be reviewed according to people's forgetting rules. In this way, students can make good arrangements to study every day without parental supervision, and at the same time do not have to hurry up and down in the examination, so that students can also spare time to review other subjects. And at the end of one semester, teachers can observe the progress of each student according to the learning records of the students by Box Fish APP, thus rewarding the students. This method can greatly improve the learning enthusiasm of the students.

\section{CONCLUSION}

The combination of information technology and Junior Middle School English vocabulary teaching can provide a lot of development space for Junior Middle School English teaching which is also conducive to improve the level of Junior Middle School English education. At the same time, the use of information technology can improve students' interest in learning and teachers' teaching level to a certain extent. And the use of Box Fish APP in English vocabulary teaching can make up for some shortcomings in traditional teaching. From the teacher's point of view, the teacher can grasp the students' learning situation in time through the Box Fish APP, so that the class can be planned well in advance. Students 
use Box Fish APP to learn before class. Teachers can teach better in class. At the same time, teachers can also pay attention to the development of students' oral pronunciation. For students, the use of intelligent learning APP meets the individual needs of students. Students can not only design teaching according to their own situation, but also make up for their weak foundation for students. And it can help them develop and know more extracurricular culture better. Students will not feel bored when using Box Fish APP, because it is vivid and rich, and enables students to experience learning in happiness. Secondly, the students insist on using Box Fish APP to clock in every day, so that the students form a habit of learning for a long time. Anyway, I believe that with the rapid development of science and technology, the use of smart APP will make the development of education better and better.

With the combination of modern information technology and primary and secondary education, learners can communicate and interact with teachers and students without time and space restrictions by using smart phones to learn. In interactive whiteboard and multimedia slide show, the developing network and software technology provide more and more learning platforms and software for mobile learning. Moreover, the international education community has long proposed to encourage students to bring their own devices, such as smart phones and tablets, into the classroom as early as 2010. Mobile learning based on smart phones started late in our country and has not been widely recognized by society, schools, teachers, parents and students. The corresponding teaching design and teaching software are relatively scarce, and the existing teaching contents are also lack of interest and attraction. All these have become obstacles to the practice of mobile learning based on smart phones. Therefore, the combination of information technology and teaching in our country still has much room for development.

\section{REFERENCES}

[1] Brooke Dinsmore. (2019). Contested affordances: teachers and students negotiating the classroom integration of mobile technology. Taylor journal, (6), 83-85.

[2] Gulcin Nagehan Sarica \& Nadire Cavus. (2009). English New trends in 21st Century English learning. Elsevier Journal, (1), 46-48.

[3] Liviu Constantin Stan. (2014). Online Teaching Technique in Maritime Learning Process. Elsevier Journal, (116), 92-95.

[4] Lv Wanlu(2017). Application research of box fish APP in junior middle school English teaching. Tianjin Normal University. 20-24.

[5] O'Connor Siobhan \& Hubner Ursula \& Shaw Toria \& Blake Rachelle \& Ball Marion. (2017). Time for TIGER to ROAR! Technology Informatics Guiding Education Reform. Nurse education today, (14), 56-58.

[6] Pimanmas Ninsuwana. (2015). The Effectiveness of Teaching English by Using Reading Aloud Technique towards EFL Beginners. Procedia-Social and Behavioral Sciences, (06), 61-64.

[7] Wu Su Fan. Research on the application of smart phone APP in English teaching -- taking English writing class as an example. Journal of Mudanjiang University, (12), 35-37.

[8] Zhang jijun, li jianzhen. ( 2010). In mobile learning mode -- a new way of independent learning. Software guide, (8), 21-23.

Ling Wang was born in Jian, China in1967. She received her bachelor of Art degree in linguistics from Jiangxi Normal University, China in 1995.

She is currently an associate professor in the School of Foreign Languages, Nanchang Normal University, Nanchang, China. Her research interests include cross-cultural teaching and teaching methods.

Prof. Wang is a member of the Chinese Association of Foreign Language Teacher. 